Research Paper

\title{
Comparison of Treatment Modalities for Locally Advanced Gastric Cancer: A Propensity Score Matching Analysis
}

\author{
Jianglong Han ${ }^{1 *}$; Zhihua Nie ${ }^{2,3^{*}}$; Ping Li1,2; Hongwei Shi ${ }^{1}$; Shijie Wang1; Qin Li1; Rui Zhang1; Yunfeng Qiao1; \\ Kejie Huang1; Zhenming $\mathrm{Fu}^{1 凶}$ \\ 1. Cancer Center, Renmin Hospital of Wuhan University, Wuhan, 430060, China \\ 2. Department of Radiation and Medical Oncology, Zhongnan Hospital of Wuhan University, Wuhan, 430071, China \\ 3. Taikang Tongji (Wuhan) Hospital, Wuhan, 430050, China \\ * These authors contributed equally to this work. \\ $\triangle$ Corresponding author: Prof. Zhenming Fu, Cancer Center, Renmin Hospital of Wuhan University, Wuhan, China, 430060. E-mail: \\ davidfuzming@whu.edu.cn. Tel: +086-18986199927
}

(C) The author(s). This is an open access article distributed under the terms of the Creative Commons Attribution License (https://creativecommons.org/licenses/by/4.0/). See http:/ /ivyspring.com/terms for full terms and conditions.

Received: 2019.10.11; Accepted: 2020.04.15; Published: 2020.05.18

\begin{abstract}
Background: A consensus regarding optimum treatment strategies for locally advanced gastric cancer (LAGC) has not yet been reached. We aimed to evaluate the efficacy of various treatment modalities for LAGC and provided clinicians salvage options under real-world situation.

Methods: Medical charts of patients with LAGC who underwent radical resection plus adjuvant chemotherapy or chemoradiotherapy from July 2003 to December 2014 were included. Validation cohort were selected from SEER database between 2004 and 2014. Kaplan-Meier and Cox proportional hazardous models were used to evaluate the overall survival (OS), cancer-specific survival (CSS), and disease-free survival (DFS). Propensity score matching (PSM) was used to adjust for potential baseline confounding.

Results: A total of 350 patients were included and divided into DI dissection plus chemotherapy group (DICT, $n=74$ ), D1 dissection plus adjuvant chemoradiotherapy group (DICRT, $n=69$ ), D2 dissection plus adjuvant chemotherapy group (D2CT, $\mathrm{n}=134)$, and D2 dissection plus adjuvant chemoradiotherapy group (D2CRT, $\mathrm{n}=73$ ). PSM identified 50 patients in each group. After PSM, better DFS (P for D2CRT vs. DICT, DICRT, and D2CT was $0.001,0.006$, and 0.001 , respectively) and OS (P for D2CRT vs. DICT, DICRT, and D2CT was $0.001,0.011$, and 0.022 , respectively) were found for the D2CRT group (mean, OS $=110.7$ months, DFS $=95.2$ months) than the other groups. Similar findings were further validated in the Surveillance, Epidemiology, and End Results database (SEER) cohort. In addition, patients in the DICRT group achieved similar survival outcomes to those in the D2CT group (mean OS, 72.8 vs. 59.1 months, $P=0.86$; mean DFS, 54.4 vs. 34.1 months, $P=0.460$ ).

Conclusions: The results of the study indicated the better role for D2CRT in treating the LAGC, meanwhile, the patients treated with DICRT might achieve similar survival as that of D2CT patients.
\end{abstract}

Key words: Gastric cancer, D1 dissection, D2 dissection, Adjuvant therapy, Propensity score matching, SEER

\section{Introduction}

Gastric cancer is the fifth most common malignancy and the third leading cause of cancer-related death worldwide [1]. East Asia, including China, Japan, and Korea, has the highest incidence rates of gastric cancer. In 2014, 410,400 new stomach cancer cases and 293,800 cancer-related deaths were estimated to have occurred in China [2].

Adequate radical resection with adjuvant therapies may be the possible curative therapy for locally advanced gastric cancer (LAGC). However, the 
preferred treatment for LAGC differs by geographical region. The recommended adjuvant therapies are perioperative chemotherapy or postoperative chemoradiotherapy in the USA and some other parts of the world [3-5], whereas the postoperative chemotherapy is preferred in Japan and South Korea $[6,7]$. Clearly, there is no universally accepted optimal strategy for LAGC treatment.

For lymph node dissection, D2 dissection is generally recommended because of its lower recurrence and cancer-related death rates than D1 dissection [8, 9]. However, D2 dissection may be associated with higher postoperative mortality and morbidity [10]. In addition, the more complicated surgical technique and prolonged operation time make D2 dissection most commonly practiced in high-volume centers with experienced surgeons [9, 11, 12]. In developing areas such as rural China, D2 dissection cannot be performed universally or is practiced in a nonstandard way due to inadequate surgical resources [13]. In Western countries, D1 or even D0 resections are still often performed for various reasons [14, 15]. Under these circumstances, salvage treatments should be sought after an inadequate lymphadenectomy. The advancement in adjuvant therapies may provide opportunities to compensate, at least partially, for the defects of imperfect lymphadenectomy. Currently, various therapeutic strategies are used in the treatment of LAGC, including but not limited to D1 dissection plus adjuvant chemotherapy (D1CT), D1 dissection plus adjuvant chemoradiotherapy (D1CRT), D2 dissection plus adjuvant chemotherapy (D2CT), and D2 dissection plus adjuvant chemoradiotherapy (D2CRT).

It is recommended in China that D2 dissection should be pursued in the treatment of LAGC [16]; thus, a randomized controlled trial (RCT) containing multiple control arms of other resection modalities might be inappropriate. Therefore, we compared the efficacy among various therapeutic strategies which were currently used in the treatment of LAGC in a retrospective cohort, through conventional and propensity score matching (PSM) approaches.

\section{Participants and Methods}

\section{Participants}

The study was performed at an affiliated Hospital of Wuhan University (Hubei, China), the Institutional Review Board approval was not required according to the institution policy because the study is a retrospective medical chart review without direct patient contact. Additional individual consents for this analysis was not needed. Medical records of newly diagnosed LAGC patients with radical operation plus adjuvant therapies in the affiliated hospital between July 2003 and December 2014 were collected. Eligible patients had to fulfill the following criteria: age between 20 years old and 75 years old, histologically confirmed gastric adenocarcinoma, R0 gastrectomy with D2 (en bloc with the N1 and N2 lymph nodes and a minimum of 15 lymph nodes examined) or D1 dissection, no evidence of gross peritoneal seeding and metastasis, stage IB-IIIC (as defined by the American Joint Committee on Cancer staging manual, 7th edition) [17], and having received at least 2 cycles of either adjuvant or concurrent chemotherapy. All patients had adequate hematologic, hepatic, and renal function tests. Excluded from the study were patients who were: T1N0M0 disease; Siewert type I/II or gastro-esophageal junction disease; no radical gastrectomy; non-R0 resection, and with distant metastases. Pretreatment evaluations consisted of medical history assessment, physical examination, hematologic and biochemical analyses, tumor marker evaluations, electrocardiography, and computed tomography (CT) of the thorax, abdomen (contrast enhanced), and pelvis. As a result, a cohort of 350 cases was included in the current study. According to the types of treatment modalities, patients were categorized into four groups, i.e., D1CT group, D1CRT group, D2CT group, and D2CRT group (74 cases, 69 cases, 134 cases, and 73 cases, respectively).

Furthermore, we aimed to validate our findings in Surveillance, Epidemiology, and End Results (SEER) database, which covered approximately 34.6\% of the US population [18]. Eligible participants were included according to the inclusion criteria in our study (Figure 1), and the selected patients were categorized into D1CT group, D1CRT group, D2CT group, and D2CRT group (457 cases, 929 cases, 844 cases, and 1,338 cases, respectively).

\section{Treatment}

All patients underwent radical resection with lymph node dissection. In this study, radiotherapy regimens were conformal radiotherapy or intensity-modulated radiotherapy (IMRT). All patients were treated following a standard postoperative CCRT protocol which has been previously described in a phase II trial [19]. For postoperative chemotherapy, the standard regimen for most patients was FOLFOX4 $\left(85 \mathrm{mg} / \mathrm{m}^{2}\right.$ oxaliplatin intravenous [IV] infusion on day $1 ; 2$ hours IV infusion of $200 \mathrm{mg} / \mathrm{m}^{2}$ leucovorin [LV] on days 12; $400 \mathrm{mg} / \mathrm{m}^{2} 5$-fluorouracil [5-FU] IV bolus on days 1-2, followed by 22-hour continuous IV infusion of $600 \mathrm{mg} / \mathrm{m}^{2}$-FU on days 1-2 every two weeks). 
Patients in the concurrent chemoradiation group received two cycles of FOLFOX4, subsequently, they received radiotherapy ( 45 Gy at 1.8 Gy per fraction, 5 fractions per week for 5 weeks) with a $5-\mathrm{FU} / \mathrm{LV}$ regimen $\left(400 \mathrm{mg} / \mathrm{m}^{2} 5\right.$-FU IV bolus $+20 \mathrm{mg} / \mathrm{m}^{2} \mathrm{LV} \mathrm{IV}$ bolus per day on the first 4 and the last 3 days of radiotherapy), followed by additional cycles of FOLFOX4. However, in this study, mFOLFOX6, CAPOX, FOLFORI, and 5-FU IV plus another chemotherapeutic drug (such as mitomycin C, cisplatin) were also allowed for chemotherapy. Tegafur, capecitabine, or 5-FU IV regimen was also allowed for concurrent chemoradiotherapy. (Table S1)

\section{Follow-up and toxicity}

Follow-up after completion of treatment consisted of visits every three months for the first two years, then every six months for the following three years, and annually thereafter. Documented disease progression was defined according to physical examination and/or imaging or biopsy confirmation. New lymph nodes measuring over $1 \mathrm{~cm}$ on CT or MRI in the short axis diameter were considered malignant. Acute toxicities, measured from the initiation of treatment to 90 days after completion, were graded using the National Cancer Institute's Common Terminology Criteria for Adverse Events version 3.0 [20]. Adverse effects were categorized as hematologic adverse events, gastrointestinal adverse events, liver toxicity, and other toxicity in this study.

\section{Statistical analysis}

PSM is a tool to reduce selection bias in nonrandomized studies [21]. Propensity score with nearest neighbor matching was performed in our study to reduce the selection bias and ensure baseline balance among treatment groups, using the calipers equal to 0.05 of the standard deviation of the logit of the propensity score. Standardized differences were computed for every two groups, and the median standardized difference was utilized to show whether the distribution among groups reached balance [22]. The covariates selected for matching were based on prior literature reports, known clinically prognostic factors, and availability in medical records. Selected

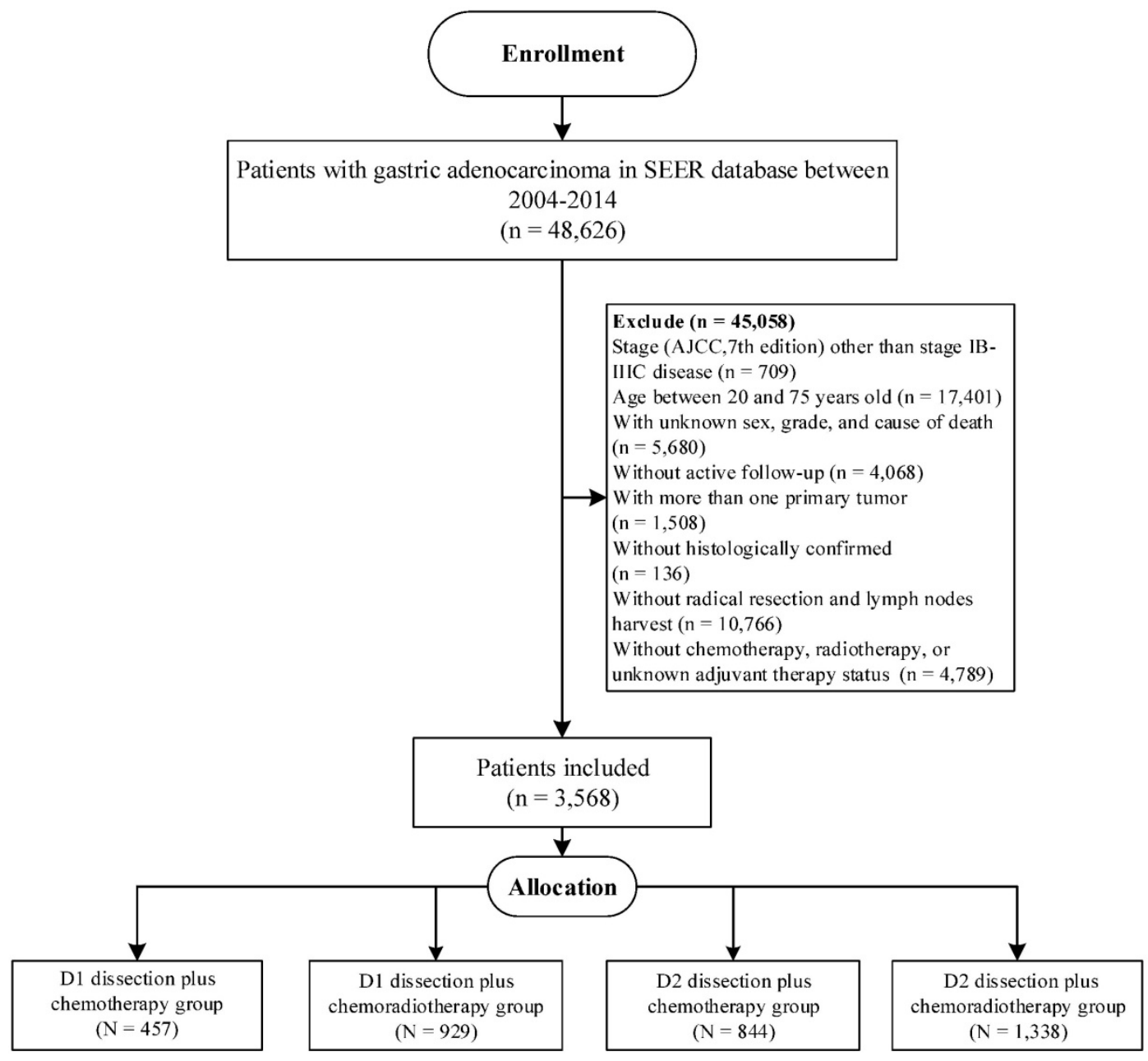

Figure 1. The flowchart of SEER validation group selection. 
variables included age, gender, tumor location, differentiated degree, metastatic lymph node ratio (MLR), and stage for primary cohort [23-25]. The propensity scores were generated stepwisely [26]. Variables were selected through univariate and then multivariable Cox regression models. Variables remained significant in the final model were selected to generate propensity scores. The distribution among groups was considered well-balanced if the standardized difference of variables $<10 \%$ [22].

The primary endpoints were overall survival (OS) and disease-free survival (DFS) for primary cohort; the endpoints were OS and cancer-specific survival (CSS) for validation cohort. Treatment-related toxicity was the secondary endpoint for primary cohort. OS was defined as the time from start of treatment to death from any cause; DFS was calculated from the date of surgery to the date of first disease progression (locoregional recurrence or metastasis). Locoregional recurrence was defined as any relapse at the remnant stomach, anastomosis site, tumor bed, or regional lymph nodes; CSS was defined as the time from treatment to death related to LAGC. Frequencies and proportions, as well as means were reported for categorical and continuous variables, respectively. General linear models or $\chi^{2}$-test were performed to compare the distribution of baseline characteristics. The Kaplan-Meier method was used for estimating survival curves. The Cox proportional hazardous model was performed to evaluate the hazard ratio $(\mathrm{HR})$ and $95 \%$ confidence interval (CI) of the associations of survival with each clinical factor. $P \leq$ 0.05 (2-sided probability) was considered statistically significant, $P \leq 0.008$ (2-sided probability) was considered statistically significant for pairwise comparison adjusted by Bonferroni method. All analyses were conducted using SAS 9.4 (SAS Institute, Cary, NC) and R software (Version 3.5.3).

\section{Results}

\section{Baseline characteristics}

Table 1 and Table 2 describe the baseline characteristics and survival outcomes before and after matching, respectively. From July 2003 to December 2014, a total of 350 patients treated with D1CT ( $\mathrm{n}=$ $74)$, D1CRT $(\mathrm{n}=69), \mathrm{D} 2 \mathrm{CT}(\mathrm{n}=134)$, and D2CRT $(\mathrm{n}=$ 73) were identified. After PSM, 50 patients in each group were left. The study was mainly carried out in males (> 60\%), patients with stage III $(>60 \%)$ and poorly differentiated $(>70 \%)$ gastric adenocarcinoma. Slightly more elderly patients $(56 \%)$ than young patients were included in the study. Table 3 demonstrates the baseline characteristics for SEER cohort. In line with the primary cohort, old males (> $70 \%)$ with advanced stage $(>50 \%)$ and poorly differentiated adenocarcinoma $(>70 \%)$ were included in validation group. Moreover, white patients accounts for $62.5 \%$ in the SEER cohort. Figure 2 shows the standardized difference among primary cohort before and after PSM. Although age and differentiation degrees for all groups were similar and only changed slightly after PSM, the standardized difference was still less than $10 \%$. The standardized difference of other variables was less than $10 \%$ after PSM, and all variables were balanced after PSM.

\section{Survival analysis}

Since the median survival in many treatment groups has not yet been reached, we present the means of survival months. As shown in the Table 1 and Table 2, 194 disease progression events occurred (52 in D1CT, 34 in D1CRT, 86 in D2CT, 22 in D2CRT groups, respectively) before matching, the lowest recurrence rate was observed in D2CRT group (30.1\% in D1CRT vs. $64.2 \%$ in D2CT, $49.3 \%$ in D1CRT, and $70.3 \%$ in D1CT, $P<0.05$ for D2CRT vs. D1CT, D1CRT, and $\mathrm{D} 2 \mathrm{CT}$, respectively). After matching, the recurrence rate remained lowest in the D2CRT group (32.0\% vs. $64.0 \%$ in D2CT, $62.0 \%$ in D1CRT, and $66.0 \%$ in D1CT, respectively).

Better DFS were found in the D2CRT group compared with other groups both before matching (mean DFS for D1CT, D1CRT, D2CT, and D2CRT was 33.4 months, 71.3 months, 46.3 months, and 95.8 months, respectively; $P$ for D2CRT vs. D1CT, D1CRT, and D2CT was 0.001, 0.063, and 0.001, respectively), similar results were also observed after matching (Figure 3B, 3D). The DFS between D1CRT and D2CRT groups became significantly different after matching $(P=0.006)$. Similar outcomes were found for OS among these groups before matching, however, the pairwise comparison among D1CRT, D2CT, and D2CRT in OS (mean OS for D2CRT, D1CRT, and D2CT was 110.7 months, 72.8 months, and 59.1 months; $P$ for D2CRT vs. D1CRT, and D2CT was $0.011,0.022$, respectively) showed no difference after matching (Figure 3A, 3C). Furthermore, similar results were observed in the validation cohort, highest OS ( $P$ for D2CRT vs. D1CT, D1CRT, and D2CT was $<0.001,0.001$, and 0.002, respectively) and CSS ( $P$ for D2CRT vs. D1CT, D1CRT, and D2CT was <0.001, 0.012 , and 0.002 , respectively) were seen in the D2CRT group (Figure 3E, 3F). Multivariate analysis consistently indicated that D2CRT was a positive prognostic factor for both OS (HR and $[95 \% \mathrm{CI}]$ for D1CT, D1CRT, and D2CT vs. D2CRT was 3.3 [1.6-6.9], 2.4 [1.1-5.1], and 2.3 [1.1-5.0]) and DFS (HR and [95\% CI] for D1CT, D1CRT, and D2CT vs. D2CRT was 
2.7 [1.5-4.9], $2.1[1.1-3.9]$, and 2.9 [1.6-5.3]), and the high MLR $(P<0.05)$ was associated with elevated risk of $\mathrm{OS}(\mathrm{HR}=1.5,95 \% \mathrm{CI}=1.0-2.3)$ and CSS $(\mathrm{HR}=1.63$, $95 \% \mathrm{CI}=1.2-2.2)($ Table 4$)$. Moreover, the validation group also revealed the positive prognostic role of D2CRT and lower MLR for both OS and CSS (Table S2 and Figure S1).

Table 1. Baseline characteristics and survival outcomes of study groups before matching.

\begin{tabular}{|c|c|c|c|c|c|c|c|}
\hline \multirow[t]{2}{*}{ Characteristic } & D1CT & D1CRT & $\mathrm{D} 2 \mathrm{CT}$ & D2CRT & $P 1$ & $P^{1}$ & $P 1$ \\
\hline & $(\mathrm{n}=74)$ & $(n=69)$ & $(n=134)$ & $(\mathrm{n}=73)$ & D1CT vs. D2CRT & D1CRT vs. D2CRT & D2CT vs. D2CRT \\
\hline Age (years) & . & & & & 0.67 & 0.53 & 0.37 \\
\hline$<55$ & $32(43.2 \%)$ & $31(44.9 \%)$ & $62(46.3 \%)$ & $29(39.7 \%)$ & & & \\
\hline$\geq 55$ & $42(56.8 \%)$ & $38(55.1 \%)$ & $72(53.7 \%)$ & $44(60.3 \%)$ & & & \\
\hline Gender & & & & & 0.096 & 0.78 & 0.25 \\
\hline Male & $56(75.7 \%)$ & $45(65.2 \%)$ & $95(70.9 \%)$ & $46(63.0 \%)$ & & & \\
\hline Female & $18(24.3 \%)$ & $24(34.8 \%)$ & $39(29.1 \%)$ & $27(37.0 \%)$ & & & \\
\hline Location & & & & & 0.96 & 0.44 & 0.54 \\
\hline Upper stomach & $21(28.4 \%)$ & $24(34.8 \%)$ & $32(23.9 \%)$ & $21(28.8 \%)$ & & & \\
\hline Middle/Lower stomach & $53(71.6 \%)$ & $45(65.2 \%)$ & $102(76.1 \%)$ & $52(71.2 \%)$ & & & \\
\hline Differentiation degree & & & & & 0.38 & 0.56 & 0.92 \\
\hline Poor differentiation & $52(70.3 \%)$ & $50(72.5 \%)$ & $102(76.1 \%)$ & $56(76.7 \%)$ & & & \\
\hline Well differentiation & $22(29.7 \%)$ & $19(27.5 \%)$ & $32(23.9 \%)$ & $17(23.3 \%)$ & & & \\
\hline Stage & & & & & 0.003 & 0.099 & 0.96 \\
\hline IB-IIB & $40(54.1 \%)$ & $30(43.5 \%)$ & $40(29.9 \%)$ & $22(30.1 \%)$ & & & \\
\hline III & $34(45.9 \%)$ & $39(56.5 \%)$ & $94(70.1 \%)$ & $51(69.9 \%)$ & & & \\
\hline \multicolumn{8}{|l|}{ Survival outcomes } \\
\hline Patients with recurrence & $52(70.3 \%)$ & $34(49.3 \%)$ & $86(64.2 \%)$ & $22(30.1 \%)$ & $<0.001$ & 0.020 & $<0.001$ \\
\hline \multicolumn{8}{|l|}{ Disease-free survival (DFS) } \\
\hline Mean, months & 33.4 & 71.3 & 46.3 & 95.8 & $<0.001$ & 0.063 & $<0.001$ \\
\hline 3-year DFS & $40.5 \%$ & $59.4 \%$ & $41.8 \%$ & $71.2 \%$ & $<0.001$ & 0.16 & $<0.001$ \\
\hline 5-year DFS & $32.4 \%$ & $52.2 \%$ & $37.3 \%$ & $69.9 \%$ & $<0.001$ & 0.02 & $<0.001$ \\
\hline \multicolumn{8}{|l|}{ Overall survival (OS) } \\
\hline Mean, months & 48.1 & 81.6 & 68.3 & 113.2 & 0.001 & 0.048 & 0.001 \\
\hline 3-year OS & $71.6 \%$ & $71.0 \%$ & $67.2 \%$ & $83.6 \%$ & 0.083 & 0.074 & 0.011 \\
\hline 5-year OS & $55.4 \%$ & $63.8 \%$ & $59.7 \%$ & $80.8 \%$ & 0.001 & 0.023 & 0.002 \\
\hline
\end{tabular}

Table 2. Baseline characteristics and survival outcomes of study groups after matching 1

\begin{tabular}{|c|c|c|c|c|c|c|c|}
\hline \multirow[t]{2}{*}{ Characteristic } & $\mathrm{D} 1 \mathrm{CT}$ & D1CRT & $\mathrm{D} 2 \mathrm{CT}$ & D2CRT & $P^{2}$ & $P^{2}$ & $P^{2}$ \\
\hline & $(\mathrm{n}=50)$ & $(n=50)$ & $(\mathrm{n}=50)$ & $(n=50)$ & D1CT vs. D2CRT & D1CRT vs. D2CRT & D2CT vs. D2CRT \\
\hline \multicolumn{5}{|l|}{ Age (years) } & 0.55 & 0.69 & 0.42 \\
\hline$<55$ & $23(46.0 \%)$ & $23(46.0 \%)$ & $24(48.0 \%)$ & $20(40.0 \%)$ & & & \\
\hline$\geq 55$ & $27(54.0 \%)$ & $27(54.0 \%)$ & $26(52.0 \%)$ & $30(60.0 \%)$ & & & \\
\hline \multicolumn{5}{|l|}{ Gender } & 0.67 & 0.68 & 0.83 \\
\hline Male & $35(70.0 \%)$ & $31(62.0 \%)$ & $32(64.0 \%)$ & $33(66.0 \%)$ & & & \\
\hline Female & $15(30.0 \%)$ & $19(38.0 \%)$ & $18(36.0 \%)$ & $17(34.0 \%)$ & & & \\
\hline \multicolumn{5}{|l|}{ Location } & 0.51 & 0.83 & 0.51 \\
\hline Upper stomach & $13(26.0 \%)$ & $15(30.0 \%)$ & $13(26.0 \%)$ & $16(32.0 \%)$ & & & \\
\hline Middle/Lower stomach & $37(74.0 \%)$ & $35(70.0 \%)$ & $37(74.0 \%)$ & $34(68.0 \%)$ & & & \\
\hline \multicolumn{5}{|l|}{ Differentiation degree } & 0.82 & 1.0 & 0.82 \\
\hline Poor differentiation & $38(76.0 \%)$ & $37(74.0 \%)$ & $38(76.0 \%)$ & $37(74.0 \%)$ & & & \\
\hline Well differentiation & $12(24.0 \%)$ & $13(26.0 \%)$ & $12(24.0 \%)$ & $13(26.0 \%)$ & & & \\
\hline \multicolumn{5}{|l|}{ Stage } & 0.84 & 0.68 & 0.84 \\
\hline IB-IIB & $18(36.0 \%)$ & $17(34.0 \%)$ & $20(40.0 \%)$ & $19(38.0 \%)$ & & & \\
\hline III & $32(64.0 \%)$ & $33(66.0 \%)$ & $30(60.0 \%)$ & $31(62.0 \%)$ & & & \\
\hline \multicolumn{8}{|l|}{ Survival outcomes } \\
\hline Patients with recurrence & $33(66.0 \%)$ & $31(62.0 \%)$ & $32(64.0 \%)$ & $16(32.0 \%)$ & 0.001 & 0.003 & 0.001 \\
\hline \multicolumn{8}{|l|}{ Disease-free survival (DFS) } \\
\hline Mean, months & 35.4 & 54.4 & 34.1 & 95.2 & $<0.001$ & 0.006 & $<0.001$ \\
\hline 3-year DFS & $42.0 \%$ & $48.0 \%$ & $50.0 \%$ & $70.0 \%$ & 0.005 & 0.025 & 0.041 \\
\hline 5-year DFS & $38.0 \%$ & $40.0 \%$ & $40.0 \%$ & $68.0 \%$ & 0.003 & 0.005 & 0.005 \\
\hline \multicolumn{8}{|l|}{ Overall survival (OS) } \\
\hline Mean, months & 44.8 & 72.8 & 59.1 & 110.7 & $<0.001$ & 0.011 & 0.022 \\
\hline 3-year OS & $66.0 \%$ & $62.0 \%$ & $72.0 \%$ & $84.0 \%$ & 0.038 & 0.013 & 0.15 \\
\hline 5-year OS & $46.0 \%$ & $58.0 \%$ & $64.0 \%$ & $80.0 \%$ & $<0.001$ & 0.01 & 0.075 \\
\hline \multicolumn{8}{|c|}{$\begin{array}{l}\text { Abbreviation: D1CT: D1 dissection plus chemotherapy; D1CRT: D1 dissection plus chemoradiotherapy; D2CT: D2 dissection plus chemotherapy; D2CRT: D2 dissection plu } \\
\text { chemoradiotherapy; }\end{array}$} \\
\hline
\end{tabular}


${ }^{2} P$ values are derived from Chi-square test or Fisher's exact test for categorical variables, and $P$ values are derived from Log rank test for disease-free survival and Overall survival.

Table 3. Baseline characteristics and survival outcomes of SEER validation cohort, 2004-2014.

\begin{tabular}{|c|c|c|c|c|c|c|c|}
\hline \multirow[t]{2}{*}{ Characteristic } & $\mathrm{D} 1 \mathrm{CT}$ & D1CRT & $\mathrm{D} 2 \mathrm{CT}$ & D2CRT & $P^{1}$ & $P^{1}$ & $P^{1}$ \\
\hline & $(n=457)$ & $(n=929)$ & $(n=844)$ & $(n=1,338)$ & D1CT vs. D2CRT & D1CRT vs. D2CRT & D2CT vs. D2CRT \\
\hline Age (years) & & & & & 0.039 & 0.22 & 0.23 \\
\hline$<55$ & $122(26.7 \%)$ & $273(29.4 \%)$ & $248(29.4 \%)$ & $426(31.8 \%)$ & & & \\
\hline$\geq 55$ & $335(73.3 \%)$ & $656(70.6 \%)$ & $596(70.6 \%)$ & $912(68.2 \%)$ & & & \\
\hline Gender & & & & & 0.61 & 0.63 & 0.71 \\
\hline Male & $309(67.6 \%)$ & $625(67.3 \%)$ & $566(67.1 \%)$ & $887(66.3 \%)$ & & & \\
\hline Female & $148(32.4 \%)$ & $304(32.7 \%)$ & $278(32.9 \%)$ & $451(33.7 \%)$ & & & \\
\hline Race & & & & & $<0.001$ & $<0.001$ & $<0.001$ \\
\hline White & $309(67.6 \%)$ & $596(64.2 \%)$ & $554(65.6 \%)$ & $772(57.7 \%)$ & & & \\
\hline Black & $59(15.1 \%)$ & $158(17.0 \%)$ & $113(13.4 \%)$ & $190(14.2 \%)$ & & & \\
\hline Other & $79(17.3 \%)$ & $175(18.8 \%)$ & $177(21.0 \%)$ & $376(28.1 \%)$ & & & \\
\hline Differentiation degree & & & & & 0.010 & 0.090 & 0.77 \\
\hline Poor differentiation & $304(66.5 \%)$ & $648(69.8 \%)$ & $621(73.6 \%)$ & $977(73.0 \%)$ & & & \\
\hline Well differentiation & $153(33.5 \%)$ & $281(30.2 \%)$ & $223(26.4 \%)$ & $261(27.0 \%)$ & & & \\
\hline Stage & & & & & $<0.001$ & $<0.001$ & $<0.001$ \\
\hline IB-IIB & $239(52.3 \%)$ & $460(49.5 \%)$ & $360(42.7 \%)$ & $458(34.2 \%)$ & & & \\
\hline III & $218(47.7 \%)$ & $469(50.5 \%)$ & $484(57.3 \%)$ & $880(65.8 \%)$ & & & \\
\hline \multicolumn{8}{|l|}{ Survival outcomes } \\
\hline \multicolumn{8}{|c|}{ Cancer-specific survival (CSS) progression } \\
\hline Mean, months & 67.8 & 76.0 & 73.9 & 82.4 & $<0.001$ & 0.012 & 0.002 \\
\hline 3-year CSS & $49.6 \%$ & $54.5 \%$ & $56.6 \%$ & $59.9 \%$ & $<0.001$ & 0.009 & 0.12 \\
\hline 5-year CSS & $44.6 \%$ & $47.5 \%$ & $49.8 \%$ & $54.0 \%$ & $<0.001$ & 0.002 & 0.052 \\
\hline \multicolumn{8}{|l|}{ Overall survival (OS) } \\
\hline Mean, months & 60.6 & 67.5 & 67.8 & 76.5 & $<0.001$ & 0.001 & 0.002 \\
\hline 3-year OS & $45.2 \%$ & $49.7 \%$ & $53.3 \%$ & $56.9 \%$ & $<0.001$ & 0.001 & 0.066 \\
\hline 5-year OS & $39.6 \%$ & $40.7 \%$ & $46.0 \%$ & $49.8 \%$ & $<0.001$ & $<0.001$ & 0.058 \\
\hline
\end{tabular}

Abbreviation: SEER: Surveillance, Epidemiology, and End Results program; D1CT: D1 dissection plus chemotherapy; D1CRT: D1 dissection plus chemoradiotherapy; D2CT: D2 dissection plus chemotherapy; D2CRT: D2 dissection plus chemoradiotherapy;

${ }_{1} P$ values are derived from Chi-square test or Fisher's exact test for categorical variables, and $P$ values are derived from Log rank test for cancer-specific survival and Overall survival.

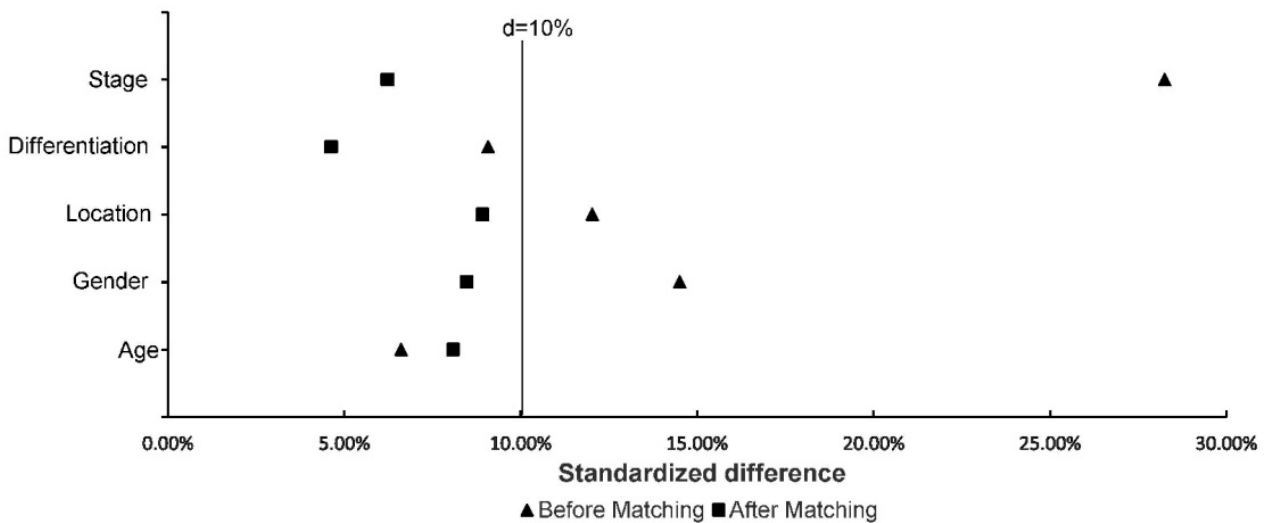

Figure 2. Standardized difference of study groups before and after matching. The distribution among groups was well balanced if the standardized difference $<10 \%$.

Table 4. Multivariate analysis of prognostic factors affecting OS and PFS of study group before and after matching.

\begin{tabular}{|c|c|c|c|c|c|c|c|c|c|c|}
\hline \multirow[t]{3}{*}{ Variables } & \multirow[t]{3}{*}{$\mathbf{N}$} & \multicolumn{4}{|c|}{ Before matching } & \multirow[t]{3}{*}{$\mathbf{N}$} & \multicolumn{4}{|c|}{ After matching ${ }^{1}$} \\
\hline & & \multicolumn{2}{|c|}{ Overall survival } & \multicolumn{2}{|c|}{ Disease-free survival } & & \multicolumn{2}{|c|}{ Overall survival } & \multicolumn{2}{|c|}{ Disease-free survival } \\
\hline & & $\mathrm{HR}(95 \% \mathrm{CI})^{2}$ & $P^{2}$ & $\mathrm{HR}(95 \% \mathrm{CI})^{2}$ & $P^{2}$ & & $\mathrm{HR}(95 \% \mathrm{CI})^{2}$ & $P^{2}$ & HR $(95 \% \mathrm{CI})^{2}$ & $P^{2}$ \\
\hline \multicolumn{11}{|c|}{ Age (<55 years as ref.) } \\
\hline$\geq 55$ years & 196 & $0.87(0.62-1.2)$ & 0.43 & $0.87(0.66-1.2)$ & 0.35 & 110 & $0.67(0.43-1.1)$ & 0.085 & $0.70(0.48-1.0)$ & 0.065 \\
\hline \multicolumn{11}{|c|}{$\operatorname{MLR}(<0.32 \text { as ref. })^{3}$} \\
\hline$\geq 0.32$ & 196 & $1.5(1.0-2.3)$ & 0.038 & $1.63(1.2-2.2)$ & 0.003 & 113 & $1.5(0.92-2.4)$ & 0.10 & $1.7(1.1-2.5)$ & 0.015 \\
\hline \multicolumn{11}{|c|}{ Stage (IB-IIB as ref.) } \\
\hline III & 218 & $1.3(0.85-1.9)$ & 0.25 & $1.4(0.98-1.9)$ & 0.080 & 126 & $1.1(0.72-1.8)$ & 0.57 & $1.3(0.89-2.0)$ & 0.18 \\
\hline \multicolumn{11}{|c|}{ Treatment (D2CRT as ref.) } \\
\hline D1CT & 69 & $2.9(1.5-5.5)$ & 0.001 & $2.8(1.7-4.8)$ & $<0.001$ & 50 & $3.3(1.6-6.9)$ & 0.001 & $2.7(1.5-4.9)$ & $<0.001$ \\
\hline D1CRT & 134 & $1.7(0.89-3.3)$ & 0.11 & $1.5(0.87-2.6)$ & 0.14 & 50 & $2.4(1.1-5.1)$ & 0.023 & $2.1(1.1-3.9)$ & 0.018 \\
\hline $\mathrm{D} 2 \mathrm{CT}$ & 73 & $2.5(1.4-4.6)$ & 0.002 & $2.8(1.8-4.5)$ & $<0.001$ & 50 & $2.3(1.1-5.0)$ & 0.029 & $2.9(1.6-5.3)$ & 0.001 \\
\hline
\end{tabular}

Abbreviation: D1CT: D1 dissection plus chemotherapy; D1CRT: D1 dissection plus chemoradiotherapy; D2CT: D2 dissection plus chemotherapy; D2CRT: D2 dissection plus chemoradiotherapy; HR: hazard ratio; MLR: metastatic lymph node ratio; CI: confidence interval. 
${ }^{1}$ Variables adjusted for matching are age, gender, tumor location, differentiated degree, MLR, and stage.

${ }^{2}$ Adjusted variables in the final model are age, MLR, stage, and treatment, hazard ratio and $P$ values are derived from Cox proportional hazardous model and Log rank test. ${ }^{3}$ MLR: Metastatic lymph node ration, defined as the ratio of positive lymph nodes in harvested lymph nodes.

\section{Adverse effect analysis}

Table S3 describes the adverse events reported during the follow-up duration. Common adverse reactions occurred in the hematological system and gastrointestinal tract. Before matching, 187 adverse events were found. Grade 3 and 4 adverse events among the four groups showed no significant difference $(P>0.05$ for every two comparison groups, data not shown). However, the incidence of grade 1 and 2 adverse events in D1CRT was significantly higher than that in the other three groups $(P<0.001$ for every two comparisons). No statistical differences were found among the other three groups. After matching, similar outcomes were found for the occurrence of grade 3 and 4 adverse events. In contrast, D2CRT had statistically fewer grade 1 and 2 events than that of D1CRT $(P=0.021)$.

\section{Subgroup analysis}

We performed a subgroup analysis according to the surgery time before and after 2011 (Figure S2). A total of 207 patients with D2 dissection (134 cases with D2CT and 73 cases with D2CRT) were enrolled; 84 cases of D2 dissection (40.6\%) were performed before 2011. Overall, the highest 5-year OS and 5-year DFS were most observed in subgroups of D2CRT. Compared with D2CRT, D2CT had significantly reduced OS and DFS for patients who received surgery before 2011 both before and after matching. For patients who received operation after 2011, the DFS of the D2CT groups were significantly reduced compared with the D2CRT group before matching (D2CT vs. D2CRT, $P=0.001$ ). However, the OS between D2CT and D2CRT groups did not show significant difference after matching $(P=0.11)$.
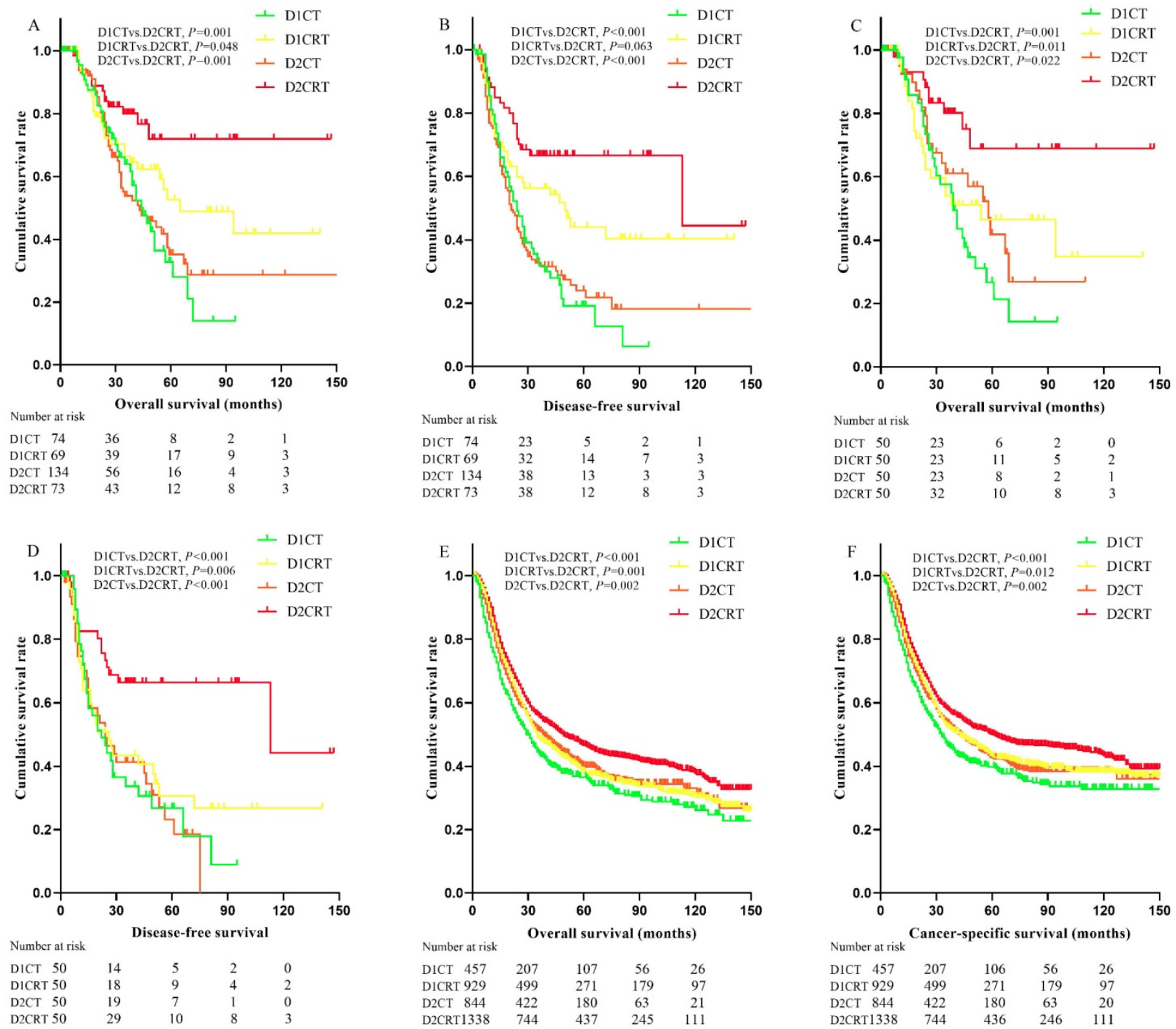
Figure 3. Kaplan-Meier curves of survival for study groups and SEER validation group. The Kaplan-Meier curves of OS (A) and DFS (B) for the study group before matching; the Kaplan-Meier curves of OS (C) and DFS (D) for the study group after matching; the Kaplan-Meier curves of OS (E) and CSS (F) in the SEER validation group. OS: overall survival, DFS: disease-free survival, CSS: cancer-specific survival, SEER: Surveillance, Epidemiology, and End Results program.

\section{Discussion}

To the best of our knowledge, the present study was the first analysis to use PSM to compare current therapeutic modalities in the treatment of patients with newly diagnosed LAGC, and SEER cohort were further used to validate our findings. In this study, we found significantly better survival outcomes in the D2CRT group than the other treatment groups. In addition, we found that the D1CRT group showed no significant differences from the D2CT group in clinical outcomes, which might indicate that radiotherapy might compensate for the defects of imperfect lymphadenectomy to a large extend for patients with LAGC.

Adjuvant therapies are used to further improve the survival and quality of life in combination with surgery. Although strategies of adjuvant therapies vary among Eastern and Western countries, the survival benefits of adjuvant therapies after resection of LAGC are universally acknowledged [11]. The Intergroup 0116 study showed that postoperative chemoradiotherapy improved both locoregional control rate and overall survival compared to surgery only [3]. In our SEER cohort, which might partially represent the American population, survival benefit was also observed in chemoradiotherapy group. However, the trial has been criticized for the inadequate surgical procedures. In fact, whether postoperative chemotherapy or chemoradiotherapy should be the preferred choice after D2 dissection of LAGC was still under debate. In East Asia, the Korean ARTIST trials found no significant differences between postoperative chemoradiotherapy and postoperative chemotherapy after D2 dissection, but an improved DFS was found in node-positive patients $[27,28]$. Furthermore, the interim result of ARTIST 2 trial indicated that postoperative chemoradiotherapy achieved similar DFS to that of chemotherapy alone in the D2-resected node-positive LAGC patients [29]. Meanwhile, a phrase III trial in China reported a significantly improved DFS in the D2CRT group [30], and similar outcomes were also observed in several previous studies [31,32]. The European CRITICS trial [33] reported that postoperative chemoradiotherapy did not improve the survival outcome compared with postoperative chemotherapy in patients who received at least D1+ dissection. In our study, the results were partially consistent with the results of the study in China in terms of DFS [30]. Furthermore, a benefit to OS was also found in our current study and in the SEER cohort. For radiotherapy, anterior-posterior opposed fields were utilized in the Korean ARTIST trials. In our study, most patients received IMRT as previously described which was dosimetrically superior to the conventional therapy used in ARTIST [19]. This might partially explain the improved OS and DFS found in the current study. We found that D1CRT obtained similar OS and DFS as that of D2CT. Although little RCTs had directly compared the efficacy of D1CRT and D2CT, an article discussed the possibility for D1CRT to replace D2 dissection [30]; it showed that adjuvant chemoradiotherapy was associated with better survival for patients with D1 dissection or R1 resection. Furthermore, 1:1 PSM among D1CT, D1CRT and D2CT groups were conducted to investigate the survival outcomes. We found that survival among D1CRT and D2CT remained similar. Furthermore, D1CRT in the validation group also showed a similar survival compared to D2CT $(\mathrm{HR}=0.96,95 \% \mathrm{CI}=0.88-1.14)$, whereas the elevated risk was observed when compared with D1CT group $(\mathrm{HR}=1.19,95 \% \mathrm{CI}=$ 1.01-1.41). These findings highlight the beneficial effects of including postoperative radiotherapy in improving survival after surgery, especially in high incidence but resources-limited areas where D2 dissection is often performed in a nonstandard way.

Furthermore, we conducted subgroup analyses by the time of surgery before or after 2011, because the understanding and implementation of the standard D2 dissection did not reach a consensus till 2011 in the hospital where the study began. It was uncertain whether the quality of earlier D2 dissections was satisfactory. Before 2011, the D2CRT group had significantly improved 5-year OS and 5-year DFS rates compared with the D2CT group both before and after matching. For patients who received surgery after 2011, D2CRT was associated with a significantly improved DFS than D2CT, but no differences were observed for OS. Thus, our results support the possibility of improving DFS for postoperative chemoradiotherapy after standard D2 dissection. The findings indicated the efficacy of D2CRT in real-life situation. Moreover, our survival outcomes among the D2CT group (5-year OS survival, $76.1 \%$ vs. $73 \%$ ) and the D2CRT group (5-year OS survival, $83.6 \%$ vs. $75 \%)$ after 2011 were comparable with those of the ARTIST trial. Therefore, although the cohort was highly selective, the quality of treatment of our patients was satisfactory. For adverse events, the number of grade 3 and 4 adverse events reported among the four groups was not significantly different or was even lower than those in other reports [33, 35]. Furthermore, the rates for grade 1 and 2 adverse 
events $(30-50 \%)$ were similar to those in reports from other Eastern countries [35, 36].

Our findings must be interpreted within the context of several limitations. Firstly, findings from the study were limited for the retrospective setting, but we recruited SEER cohort which might represent the American population to validate and extrapolate our findings. Moreover, it is inappropriate to design RCTs to compared the multiple treatment arms containing other suboptimal resection methods. Secondly, we selected the patients mainly based on completeness of clinical information. Most of the patients selected were residents of the local city and follow-ups were convenient to be performed. Patients in the study received major kinds of adjuvant treatments, these treatments might be heterogenous. However, this might be more representative of the real world to some extent. Thirdly, in the design of this study, patients who received neoadjuvant therapy were excluded since neoadjuvant therapy is not routinely performed in our institution. We found that most of the patients who received neoadjuvant therapy were first treated in local smaller hospitals before they received an operation opportunity in our hospital. This study is in no way intended to advocate for D1 dissection nor support D1CRT as an alternative to D2CT or D2CRT. Rather, we suggest that D2 dissection should be attempted whenever possible for LAGC, including in resource-limited areas. In fact, this study is intended to find practical options for oncologists under resource-limited conditions when D2 dissection is not optimally achieved.

In conclusion, we highlighted the beneficial effects of postoperative radiotherapy in current therapeutic modalities for treating LAGC. Given the lack of feasibility to design RCTs and compare the currently available modalities directly, although we suggest that D2 dissection should be attempted whenever possible, we recommend LAGC patients for postoperative radiation therapy when patients received suboptimal surgery.

\section{Abbreviations}

CI: confidence interval; D1CT: D1 dissection plus adjuvant chemotherapy; D1CRT: D1 dissection plus adjuvant chemoradiotherapy; D2CT: D2 dissection plus adjuvant chemotherapy; D2CRT: D2 dissection plus adjuvant chemoradiotherapy; DFS: disease-free survival; HR: hazard ratio; LAGC: locally advanced gastric adenocarcinoma; MLR: metastatic lymph node ratio; OS: overall survival; PSM: propensity score matching; CT: computed tomography; MRI: magnetic resonance imaging; RCT: randomized controlled trial.

\section{Supplementary Material}

Supplementary figures and tables. http://www.jcancer.org/v11p4421s1.pdf

\section{Acknowledgements}

We sincerely thank the National Cancer Institute and the SEER staff for providing this invaluable database.

\section{Funding}

This work was partially supported by the National Natural Science Foundation of China [grants $81472971,81773555]$, the funders had no role in the design of the study or the interpretation of the findings.

\section{Author Contributions}

$\mathrm{ZF}$ : guarantor of the article. $\mathrm{ZF}, \mathrm{ZN}$, and $\mathrm{JH}$ : conception/design. JH, ZN, PL, SW, QL, YQ, and HS: collection and/or assembly of data. PL, QL, HS, RZ, $\mathrm{JH}$, and $\mathrm{KH}$ : data analysis and interpretation. $\mathrm{JH}, \mathrm{ZN}$, $\mathrm{PL}$, and ZF: manuscript writing. ZF, JH, ZN, PL, HS, SW, QL, RZ, YQ, and KH: final approval of manuscript.

\section{Competing Interests}

The authors have declared that no competing interest exists.

\section{References}

1. Bray F, Ferlay J, Soerjomataram I, et al. Global cancer statistics 2018: GLOBOCAN estimates of incidence and mortality worldwide for 36 cancers in 185 countries. CA Cancer J Clin. 2018; 0: 1-31.

2. Yang L, Zheng R, Wang N, et al. Incidence and mortality of stomach cancer in China, 2014. Chin J Cancer Res. 2018; 30: 291-298.

3. Smalley SR, Benedetti JK, Haller DG, et al. Updated analysis of SWOG-directed intergroup study 0116: a phase III trial of adjuvant radiochemotherapy versus observation after curative gastric cancer resection. J Clin Oncol. 2012; 30: 2327-2333.

4. [Internet] Ajani JA, D'Amico TA, Baggstrom M, et al. NCCN Clinical Practice Guidelines in Oncology: Gastric Cancer, Version 2. 2018. https://www.ncen.org/

5. Okines A, Verheij M, Allum W, et al. Gastric cancer: ESMO clinical practice guidelines for diagnosis, treatment and follow-up. Ann Oncol. 2010; 21 (Suppl 5): S50-S54.

6. Lee JH, Kim JG, Jung HK, et al. Clinical practice guidelines for gastric cancer in Korea: an evidence-based approach. J Gastric Cancer. 2014; 14: 87-104.

7. Japanese Gastric Cancer Association. Japanese gastric cancer treatment guidelines 2014 (ver. 4). Gastric Cancer. 2017; 20: 1-19.

8. Seevaratnam R, Bocicariu A, Cardoso R, et al. How many lymph nodes should be assessed in patients with gastric cancer? A systematic review. Gastric Cancer. 2012; 15 (Suppl 1): S70-S88.

9. Songun I, Putter H, Kranenbarg EM-K, et al. Surgical treatment of gastric cancer: 15-year follow-up results of the randomised nationwide Dutch D1D2 trial. Lancet Oncol. 2010; 11: 439-449.

10. Cuschieri A, Weeden S, Fielding J, et al. Patient survival after D1 and D2 resections for gastric cancer: long-term results of the MRC randomized surgical trial. Br J Cancer. 1999; 79: 1522-1530.

11. Van Cutsem E, Sagaert X, Topal B, et al. Gastric cancer. Lancet. 2016; 388: 2654-2664.

12. Degiuli M, Sasako M, Ponti A, et al. Morbidity and mortality in the Italian gastric cancer study group randomized clinical trial of D1 versus D2 resection for gastric cancer. Br J Surg. 2010; 97: 643-649.

13. Ji J, Shan F. Opportunities and challenges for the progress of Chinese gastric cancer surgery. Chin J Digest Surg. 2018; 17: 18-21.

14. Verlato G, Giacopuzzi S, Bencivenga $M$, et al. Problems faced by evidence-based medicine in evaluating lymphadenectomy for gastric cancer. World J Gastroenterol. 2014; 20: 12883-12891. 
15. Giuliani A, Miccini M, Basso L. Extent of lymphadenectomy and perioperative therapies: two open issues in gastric cancer. World J Gastroenterol. 2014; 20: 3889-3904.

16. Laparoscopic and endoscopic group, Chinese Society of Surgery. Guidelines of laparoscopic surgery for gastric cancer. Chin J Digest Surg. 2007; 6: 476-480.

17. Edge S, Byrd D, Compton C, et al. AJCC cancer staging manual, 7th edition. New York, USA: Springer; 2010.

18. [Internet] Surveillance, Epidemiology, and End Results Program (SEER). Overview of the SEER program, 2018. https://seer.cancer.gov/about/ overview.html.

19. $\mathrm{Yu} \mathrm{H}, \mathrm{Wu} \mathrm{Q}, \mathrm{Fu} \mathrm{Z}$, et al. A new approach to delineating lymph node target volumes for post-operative radiotherapy in gastric cancer: a phase II trial. Radiother Oncol. 2015; 116: 245-51.

20. Trotti A, Colevas AD, Setser A, et al. CTCAE v3.0: development of a comprehensive grading system for the adverse effects of cancer treatment. Semin Radiat Oncol. 2003; 13: 176-181.

21. Rosenbaum PR, Rubin DB. The central role of the propensity score in observational studies for causal effects. Biometrika. 1983; 70: 41-55.

22. Austin PC. Using the standardized difference to compare the prevalence of a binary variable between two groups in observational research. Commun Stat Simul Comput. 2009; 38: 1228-1234.

23. Shiraishi N, Inomata M, Osawa N, et al. Prognostic significance of alarm symptoms in patients with gastric cancer. Brit J Surg. 2005; 92: 840-846.

24. Wang J, Dang P, Raut CP, et al. Comparison of a lymph node ratio-based staging system with the 7th AJCC system for gastric cancer: analysis of 18,043 patients from the SEER database. Ann Surg. 2012; 255: 478-485.

25. Tytgat GN, Bartelink H, Bernards R, et al. Cancer of the esophagus and gastric cardia: recent advances. Dis Esophagus. 2004, 17: 10-26

26. Brookhart MA, Schneeweiss S, Rothman KJ, et al. Variable selection for propensity score models. Am J Epidemiol. 2006; 163: 1149-1156.

27. Park SH, Sohn TS, Lee J, et al. Phase III trial to compare adjuvant chemotherapy with capecitabine and cisplatin versus concurrent chemoradiotherapy in gastric cancer: final report of the adjuvant chemoradiotherapy in stomach tumors trial, including survival and subset analyses. J Clin Oncol. 2015; 33: 3130-3136.

28. Kim Y, Park SH, Kim KM, et al. The Influence of metastatic lymph node ratio on the treatment outcomes in the adjuvant chemoradiotherapy in stomach tumors (ARTIST) trial: a phase III trial. J Gastric Cancer. 2016; 16: 105-110.

29. Park SH, Zang DY, Han B, et al. ARTIST 2: Interim results of a phase III trial involving adjuvant chemotherapy and/or chemoradiotherapy after D2-gastrectomy in stage II/III gastric cancer (GC). J Clin Oncol. 2019; 37: 4001.

30. Zhu WG, Xua DF, Pu J, et al. A randomized, controlled, multicenter study comparing intensity-modulated radiotherapy plus concurrent chemotherapy with chemotherapy alone in gastric cancer patients with D2 resection. Radiother Oncol. 2012; 104: 361-366

31. Min C, Bangalore S, Jhawar S, et al. Chemoradiation therapy versus chemotherapy alone for gastric cancer after R0 surgical resection: a meta-analysis of randomized trials. Oncology. 2014; 86: 79-85.

32. Zhou ML, Kang M, Li GC, et al. Postoperative chemoradiotherapy versus chemotherapy for R0 resected gastric cancer with D2 lymph node dissection: an up-to-date meta-analysis. World J Surg Oncol. 2016; 14: 209-217.

33. Cats A, Jansen EPM, van Grieken NCT, et al. Chemotherapy versus chemoradiotherapy after surgery and preoperative chemotherapy for resectable gastric cancer (CRITICS): an international, open-label, randomised phase 3 trial. Lancet Oncol. 2018;19: 616-628.

34. Jansen EP, Boot H, Van de velde CJ, et al. Can adjuvant chemoradiotherapy replace extended lymph node dissection in gastric cancer? Recent Results Cancer Res. 2012;196: 229-240.

35. Bang YJ, Kim YW, Yang HK, et al. Adjuvant capecitabine and oxaliplatin for gastric cancer after D2 gastrectomy (CLASSIC): a phase 3 open-label, randomised controlled trial. Lancet. 2012; 379: 315-321.

36. Lee J, Lim DH, Kim S, et al. Phase III trial comparing capecitabine plus cisplatin versus capecitabine plus cisplatin with concurrent capecitabine radiotherapy in completely resected gastric cancer with D2 lymph node dissection: the ARTIST trial. J Clin Oncol. 2012; 30: 268-273. 